\title{
Low-temperature thermodynamics of two-dimensional electron gas on disordered host-lattice
}

\author{
V.V. Slavin \\ B. Verkin Institute for Low Temperature Physics and Engineering of the National Academy of Sciences of Ukraine \\ 47 Lenin Ave., Kharkov 61103, Ukraine \\ E-mail: slavin@ilt.kharkov.ua
}

Received November 13, 2008

\begin{abstract}
Low-temperature thermodynamic properties of two-dimensional electron ensemble on ordered and disordered host-lattice is studied numerically by transfer-matrixes method. It is shown that at low temperatures and weak disordering of host-lattice sites positions change in chemical potential leads to successive transitions of the system from ordered phases (like generalized Wigner crystal) to disordered states. The ranges of stability of these crystals as the function of temperature, chemical potential and disorder parameter are established.
\end{abstract}

PACS: 05.10.-a Computational methods in statistical physics and nonlinear dynamics; 05.20.-y Classical statistical mechanics.

Keywords: Generalized Wigner crystal, thermodynamics, low-dimensional systems, disordered systems.

\section{Introduction}

The layered and low-dimensional conductors attracts significant interest of researchers. This interest caused by a number of unusual thermodynamic and kinetic properties inherited by such systems. Among these systems the conductors with Coulomb self-localization (CS) are of special interest. It is well known that CS appears when charge carriers tunneling between host-lattice sites is suppressed by their mutual Coulomb repulsing. The charge carriers becomes fully localized on host-lattice sites and the only mechanism of particles motion is jumps of electrons (or holes). As the result, the dynamics of CS systems is discrete. The exact criterion of CS is the smallness of overlapping integral, $t$, in contrast with the typical change $\delta \varepsilon \sim\left(a_{0} \bar{l}\right)^{2} \bar{\varepsilon}$ in inter-particle repulsion energy as it hops (tunnels) between the host-lattice sites. Here $a_{0}$ is mean host-lattice spacing, $\bar{l} \sim a_{0}\left(1 / c_{e}\right)^{1 / d}$ mean inter-particle distance, $\bar{\varepsilon}$ the average Coulomb energy per particle, $d$ dimension of the system and $c_{e}$ is electron concentration. As was shown in [1], in zero-temperature limit and in the case of $c_{e}<<1$, the charge carriers forms ordered structure - generalized Wigner crystal (GWC). The ground state (GS) structure of GWC is fully described in terms of one-dimensional (1D) theory developed by Hubbard [2-4]. It should be noted that GWC formation has universal nature irrespective of the potential of inter-particle repulsion details and geometry of host-lattice. Such a «lowering of dimension» leads, in particular, to rather specific zero-temperature dependence of $c_{e}$ against chemical potential, $\mu$, which is well developed fractal structure of «devil staircase» type [3].

One of the most famous examples of $2 \mathrm{D} \mathrm{GWL}$ is the so called MOSFET structure (metal-oxide-semiconductor field-effect transistor) with holes impurity band. Besides, the systems where the charge carriers are pressed out on surface by external electric field applied perpendicular to surface also belong to CS class of conductors. In this case the applied electric field plays the role of chemical potential $\mu$. Changing in $\mu$ varies electron concentratio $c_{e}$ in wide range. Besides, there are strong reasons to suggest that CS criterion $(t<\delta \varepsilon)$ can be also fulfilled in layered metalooxides of high temperature superconductor types.

Another important group is 1D CS compounds. These are, first of all, quasi-one-dimensional organic conductors [4]. Furthermore, a lot of artificially created $1 \mathrm{D}$ nano-systems systems, such as chains of quantum dots, exchanging by electrons [5], chains of metallic nano-grains with tunnel junctions (organic molecules of different types) between them [6] also belong to this group.

Is of special interest the question about an influence of disorder in host-lattice positions on low-temperature properties of these systems. This question appears naturally because the overwhelming majority of CS conductors are essentially disordered systems. For instance, in 
semiconductors MOSFET type this disorder is caused by random distribution of impurities [7,8], in some nanostructures $[5,6]$ the disorder is determined by a dispersion on tunneling junctions.

Besides analytical approaches to such system investigations (see, for example $[1-4,9,10]$ ), an interest to the numerical studies has grown rapidly during the last decade. This interest is caused by the fact that the analytical theories are mainly focused on 1D systems and based on very rough simplifying models that leads, as the result, to qualitative estimations only. At the same time just the details of the ground state structure, the exact structure of elementary excitations spectrum, the thermodynamic and kinetic properties of such systems contain the most interesting and important information. This information, in particular, casts light on the extremely significant questions about the ergodic or non-ergodic behavior of the systems, the spectrum of relaxation times etc. In this connection the methods of computer simulations like Monte-Carlo [11,12] become the most powerful and useful scientific instruments. The merits and demerits of the methods are well known. The universality and flexibility should be attributed to advantages of these methods. At the same time the difficulties accompanying the investigation of non self-averaging characteristics of the systems (for example, the configurations) are shortcomings, undoubtedly. Besides, the accuracy of calculation drops crucially with disorder growing, whereas the calculation time increase essentially. Last time new modifications of Monte-Carlo methods have appeared. First of all, this is the so-called multicanonical approach [13]. However, the questions about convergence and authenticity of the obtained results are open yet. As opposed to Monte-Carlo simulation, we propose exact computer investigation of 2D CS systems using transfer-matrix method [14]. The evident merit of this method is easy controlled accuracy. The demerit is the restriction on the system size caused by computer productivity. The matter is that the calculation time for this model is proportionate to $L 2^{3 L}$, where $L$ is linear size of the system. Analogously, the memory size, required for the calculation $\sim 2^{2 L}$.

The main goal of this paper is to study low-temperature thermodynamic properties of 2D CS systems and influence of disorder in host-lattice sites positions on these properties. Besides, even in the case of regular host-lattice the theory developed in [1] is inapplicable for concentration range $1 / 2<c_{e}<1$. The proposed method allows us to fill this gap.

\section{Hamiltonian}

We will consider rarified electron gas $\left(c_{e} \leq 1\right)$ and, thus, spin indexes are dropped (model of spin-less fermions). Besides, we will neglect tunneling of electrons between host sites. The Hamiltonian of such a system, $\mathcal{H}$, can be written as:

$$
\mathcal{H}=1 / 2 \sum_{\mathbf{r}_{i} \neq \mathbf{r}_{j}} u\left(\mathbf{r}_{i}-\mathbf{r}_{j} \mid\right) n_{\mathbf{r}_{i}} n_{\mathbf{r}_{j}}-\mu \sum_{\mathbf{r}_{i}} n_{\mathbf{r}_{i}},
$$

here $\mathbf{r}_{i}$ are coordinates of 2D host-lattice sites; independent variable $n_{i}=0,1$ is a number of electrons in $\mathbf{r}_{i}$ th host-lattice site (occupation numbers); $\mu$ is chemical potential and $u\left(\left|\mathbf{r}_{i}-\mathbf{r}_{j}\right|\right)$ a screened Coulomb potential of the inter-electron repulsion; summation is carried out over all the sites. It is clear that in the case of ordered host-lattice the vectors $\mathbf{r}_{i}$ can be written as $\mathbf{r}_{i}=\mathbf{a} k+\mathbf{b} l$, where $\mathbf{a}$ and $\mathbf{b}$ are primitive translation vectors and $k, l$ are some integer, in the case of diordered host-lattice the vectors $\mathbf{r}_{i}$ arranged randomly.

\section{Approximation}

In this paper the lattice-gas model with near-neighbor (NN) approximation is used. In spite of the simplicity, this model allows us to establish new properties of the system under consideration. Adaptation of the model demand an additional punctuality, because the long-range potential of inter-electron repulsion is cut off over the distances $a_{0}$ (we will consider squad host-lattice with interval between host-lattice sites equals $a_{0}$ ). At the same time, it is well known that the details of the $u(r)$ do not affect qualitatively on the thermodynamic characteristics $[2,3,15]$, if the following restrictions should be fulfilled: (i) $u(r)$ is monotonic, everywhere convex function, (ii) $u(r)$ diminishes faster than $r^{-1}$. $\mathrm{NN}$-approximation does not satisfied the above restrictions in full extend, but as was recently shown in the case of $1 \mathrm{D}$ systems of these sort [10], such changes in $u(r)$ lead to a weak modification of the characteristics of the system only. Besides, we will consider strong-screened Yukava-like potential with radius of interaction $R_{0} \sim a_{0}$ :

$$
u(|r|)=u_{0} \exp \left(-|r| / R_{0}\right)|r|,
$$

constant $u_{0}$ was chosen as the following: $u_{0}=\exp \left(a_{0} / R_{0}\right)$, so that $u\left(a_{0}\right)=1$ for all $R_{0}$. All the mentioned above allows us to consider the proposed model as completely adequate one.

As usual, instead of Hamiltonian (1) with random host-lattice sites positions and regular function $u(|r|)$ it is convenient to transfer randomness into potential of interaction and consider the system as an electron ensemble on regular host-lattice with random function $u(|r|)$. In such an approximation the Hamiltonian (1) has the following form:

$$
\mathcal{H}=\sum_{<i, j>}^{N} u_{i, j} n_{i} n_{j}-\mu \sum_{i}^{N} n_{i}
$$


here $N$ is the total number of host-lattice sites; all these sites are enumerated by index $i, u_{i, j}$ is random energy of interaction between the sites with numbers $i$ and $j$, sign $<i, j>$ means that the summation in first term is taken over near neighbors of $i$ th site. In the simplest case of strictly ordered squad host-lattice the Hamiltonian (2) can be easily represented in spin variables $s_{i}=2 n_{i}-1$, where $s_{i}=-1,1$.

$$
\mathcal{H}=\frac{\tilde{u}}{4} \sum_{<i, j>}^{N} s_{i} s_{j}+\frac{(2 \tilde{u}-\mu)}{2} \sum_{i}^{N} s_{i}+\frac{(\tilde{u}-\mu) N}{2} .
$$

Here $\widetilde{u}=u\left(a_{0}\right)+u\left(a_{0} \sqrt{2}\right)$. It should be stressed that in Hamiltonian (3) the inverse-symmetry is broken for all $\mu \neq 2 \widetilde{u}_{0}$. In other words, beside «external filed» $\mu$, an effective «internal» field determined by $\tilde{u}$ affects on «spins». That is why in the limit $\mu \rightarrow 0$ the ground state of (2) and (3) is vacuum-like one.

In this paper we will consider squad host lattice and, thus, $\mathbf{a}=a_{0} \mathbf{i}, \mathbf{b}=a_{0} \mathbf{j}$ (i and $\mathbf{j}$ are unit vectors in $X$ and $Y$ directions, correspondingly). It is convenient to rewrite the microscopic variables $n_{\mathbf{r}_{i}}$ as the following: $n_{\mathbf{r}_{i}} \equiv n_{k, l}$, where $\mathbf{r}_{i}=\mathbf{a} k+\mathbf{b} l(k, l$ are integer).

The interaction energies $u\left(\left|\mathbf{r}_{i}-\mathbf{r}_{i^{\prime}}\right|\right)$ we rewrite as the following:

$$
\begin{gathered}
u\left(\mid \mathbf{r}_{i}-\mathbf{r}_{i^{\prime}}\right)= \\
\left.=u\left(\mid \mathbf{a}\left(k-k^{\prime}\right)+\mathbf{b}\left(l-l^{\prime}\right)+\boldsymbol{\xi}_{k, l}-\xi_{k^{\prime}, l^{\prime}}\right) \mid\right) \equiv u\left(k, l, k^{\prime}, l^{\prime}\right) .
\end{gathered}
$$

Here $\xi_{k, l}$ and $\xi_{k^{\prime}, l^{\prime}}$ are random shift vectors of the correponding host-lattice sites. The module of $\xi$ has normal distribution law with dispersion $\sigma$ which is varied from 0 (ordered host-lattice) up to 1 (complete disorder). The angular dependence of $\xi$ is random one with uniform distribution. The Hamiltonian (2) acquires the form:

$$
\begin{gathered}
\mathcal{H}=\sum_{k=1}^{L} \sum_{l=1}^{L} \sum_{k^{\prime}=k+1}^{L+1} \sum_{l^{\prime}=l+1}^{L+1} u\left(k, l, k^{\prime}, l^{\prime}\right) n_{k, l} n_{k^{\prime}, l^{\prime}}- \\
-\mu \sum_{k=1}^{L} \sum_{l=1}^{L} n_{k, l} .
\end{gathered}
$$

Here $L$ is linear size of the system $\left(L^{2}=N\right)$. We impose toroidal boundary condition on the system. It means that

$$
n_{k, L+1}=n_{i, 1}, \quad n_{L+1, l}=n_{1, l} .
$$

Let us introduce a set of occupation number values $\alpha$-th line

$$
v_{\alpha}=\left\{n_{1, \alpha}, n_{2, \alpha}, \ldots, n_{L, \alpha}\right\} .
$$

Taking into account the toroidal boundary condition $v_{L+1}=v_{1}$. In NN-approximation $\alpha$ th line interacts with $(\alpha-1)$ th and $(\alpha+1)$ th lines only. The interaction energy of $\alpha$ th and $(\alpha+1)$ th lines, $E\left(v_{\alpha}, v_{\alpha+1}\right)$, has the form

$$
\begin{gathered}
E\left(v_{\alpha}, v_{\alpha+1}\right)=\sum_{k} u(k, \alpha, k, \alpha+1) n_{k, \alpha} n_{k, \alpha}+ \\
+\sum_{k} u(k, \alpha, k+1, \alpha+1) n_{k, \alpha} n_{k+1, \alpha+1}+ \\
\quad+\sum_{k} u(k, \alpha, k-1, \alpha+1) n_{k, \alpha} n_{k-1, \alpha+1} .
\end{gathered}
$$

The first term in (5) is interaction energy of electrons in $\alpha$ th line with vertical neighbors from $(\alpha+1)$ th line, second and third terms are corresponding energies of diagonal interactions. Besides, let us introduce $E\left(v_{\alpha}\right)$ which is energy of electron interaction in $\alpha$ th line (horizontal interaction) plus the energy produced by chemical potential $\mu$.

$$
E\left(v_{\alpha}\right)=\sum_{k} u(k, \alpha, k+1, \alpha) n_{k, \alpha} n_{k+1, \alpha}-\mu \sum_{k, l} n_{k, l} .
$$

The corresponding transfer-matrixes $\hat{P}_{\alpha}[14]$ have the form:

$$
<v_{\alpha}\left|\hat{P}_{\alpha}\right| v_{\alpha+1}>=\mathrm{e}^{-\frac{1}{T}\left(E\left(v_{\alpha}, v_{\alpha}+1\right)+E\left(v_{\alpha}\right)\right)} .
$$

Here $T$ is the temperature. It should be noted again that the proposed method allows us to include into consideration both vertical (horizontal) and diagonal $\mathrm{NN}$ interactions.

The big sum is

$$
z=\sum_{\left\{n_{i}\right\}} \mathrm{e}^{\left(\frac{-\mathcal{H}}{T}\right)}=\operatorname{Spur}\left(\prod_{\alpha=1}^{\mathrm{N}} \hat{P}_{\alpha}\right),
$$

the summation is carried out over microscopic states $\left\{n_{i}\right\}$. Thermodynamic potential

$$
f(T, \mu)=-\frac{1}{N} T \ln (Z)
$$

has been calculated numerically. For algorithm testing the dependence $f(T, 0)$ has been calculated for microscopic variables $n_{i}=-1,1$ (Ising model) and ordered host-lattices $6 \times 6,8 \times 8$ and $10 \times 10$. The result is presented in Fig. 1. The exact solution (dashed curve) is plotted for comparison. One can see good agreement even for rather small host-lattices.

\section{Low temperature thermodynamics}

As was shown in [1] the ground state configuration of 2D lattice electron gas on ordered host-lattice is fully described in term of 1D Hubbard's theory [4]. It means, in particular, that for any fixed $\mu$ the ground state configuration is periodic structure (crystal) composed of one or two sort of electron bands. Both these bands are parallel, infinite in one direction and have fixed shift vector in other direction. If the concentration of the bands is of the form $c_{e}(\mu)=1 / q, q=2,3, \ldots$, then the only one sort of electron bands appear in the GS structure (see, for example, 


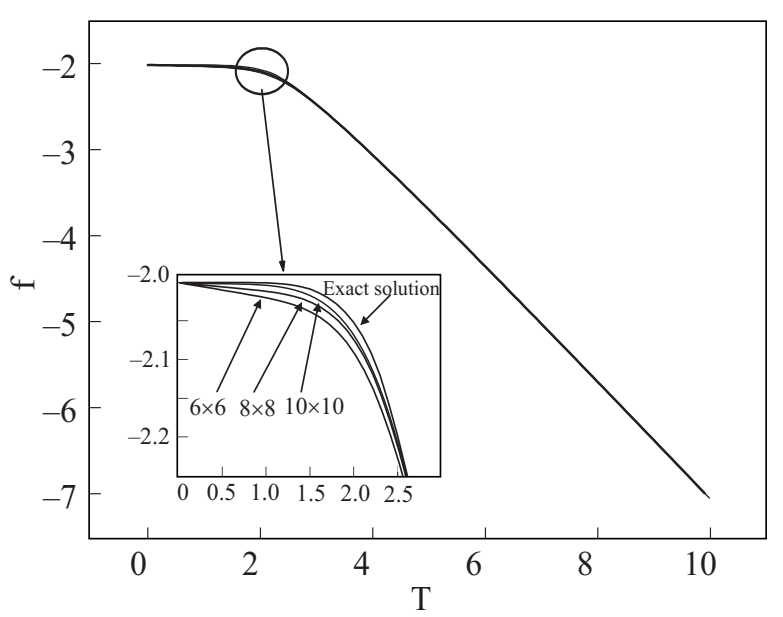

Fig. 1. The results of calculation $f(T, \mu=0)$ for regular Ising model $\left(n_{i}=-1.1\right)$. Host-lattice sizes are: $6 \times 6,8 \times 8$ and $10 \times 10$. In addition, the curve corresponding to exact solution is presented. The region of maximal deviation numerical and exact solutions is presented on insert.

Fig. 2,a). Otherwise, two types of bands form the ground state structure (see Fig. 2,b). The mutual arrangement of these bands can be calculated on the base of simple formula [2]:

$$
r_{i}=a_{0}\left[i / c_{e}\right]
$$

This formula was obtained for 1D systems of this sort. It describes the mutual arrangement of the electrons with concentration $c_{e}$ over 1D host-lattice with period $a_{0}$. Sign [...] means an integer part, $r_{i}$ is the position (coordinate) of $i$ th electron. In 2D case $r_{i}$ has sense of the position of the electron «band» with number $i$. As mentioned above, one of the most interesting consequence of such structure of the GS is zero-temperature dependence $c_{e}(\mu)$ which is well-developed fractal structure of devil-stair type [2,3].

In this connection it is reasonable to start the investigation of 2D systems from low-temperature dependence
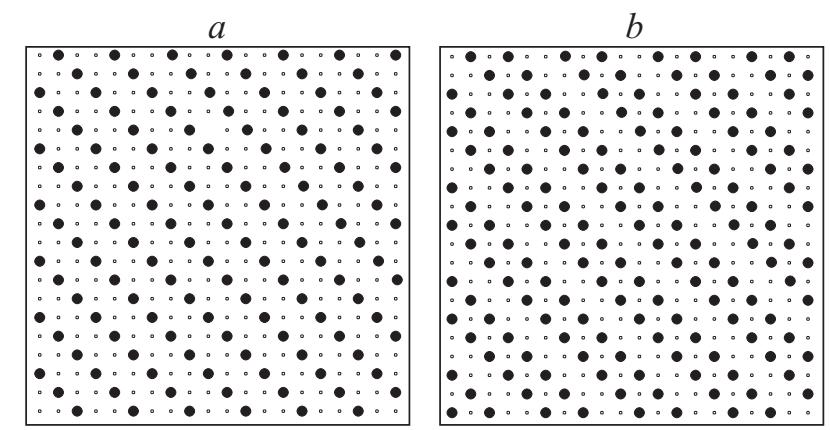

Fig. 2. Examples of 2D GWL corresponding to different electron concentrations $c_{e} \cdot c_{e}=1 / 3(a), c_{e}=2 / 5(b)$. $c_{e}(\mu)$. It's also interesting and important, because the results of theory [1] are applicable for concentration region $c_{e}<1 / 2$ only. At the same time the proposed NN approximation allows us to investigate the thermodynamic properties of the system under just in the region $c_{e} \leq 1$.

As far as $c_{e}=\frac{1}{N} \sum_{i=1} n_{i}$, the low-temperature dependence $c_{e}(\mu)$ can be calculated as the following:

$$
c_{e}(\mu)=-\left.\frac{\partial f(T, \mu)}{\partial \mu}\right|_{T \rightarrow 0} .
$$

The results of calculation $c_{e}(\mu)$ for $\sigma=0$ (ordered host-lattice), $a_{0}=1$ and fixed temperature $T=10^{-3} u\left(a_{0}\right)$ are presented in Fig. 3. One can see stair-like dependence with the stairs, corresponding to rational electron concentrations. In the limiting case of $R_{0}<<a_{0}$, when the only vertical and horizontal $\mathrm{NN}$ interactions are taken into considerations, the stairs with $c_{e}=1 / 2$ and 1 (curve $a$ ) appear. Taking into account diagonal NN interactions leads to appearance of additional stairs with $c_{e}=1 / 4$ and $3 / 4$ (curve $b$ ). It means that the effective lowering of the dimension and generalized Wigner crystal (GWC) formation, discovered in [1] preserves in the region $1 / 2 \leq c_{e} \leq 1$.

An influence of the disorder in host-lattice positions, $\sigma$ on $c_{e}(\mu)$ dependence is presented in Fig. 4. The calculations were carried out for host-lattice $10 \times 10$, $T=10^{-3} u\left(a_{0}\right)$ and $R_{0}=1$. Besides, an additional averaging of the results over 10 random realizations were carried out. The dependence of typical fluctuation $\rho$ as the function of realization number $N_{0}$ is presented in Fig. 5 . As it seen from the figure, an increase of $N_{0}$ over 10 does not effect on $\rho$ almost.

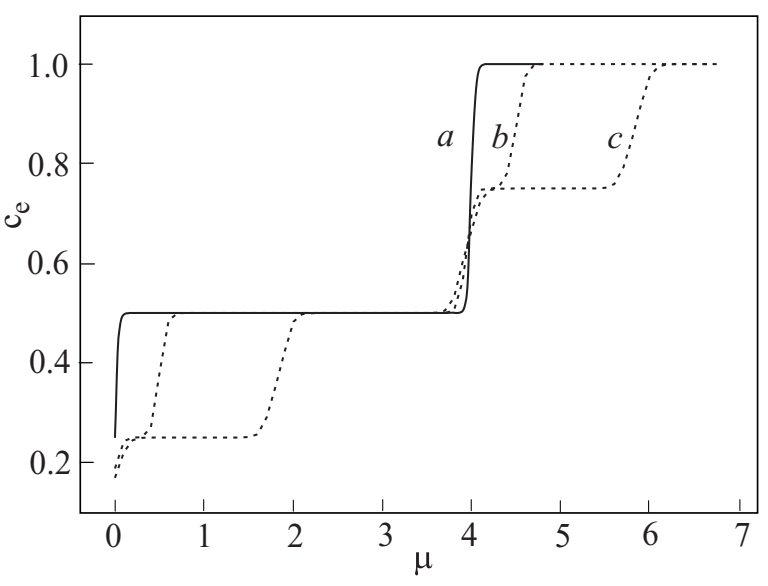

Fig. 3. The dependence $c_{e}(\mu)$ for ordered host-lattice $(\sigma=0)$ and different values of $R_{0}>T=10^{-3} u\left(a_{0}\right)$, host-lattice size is $10 \times 10 . R_{0}: 10^{-3}(a), 0.25(b), 1(c)$. 


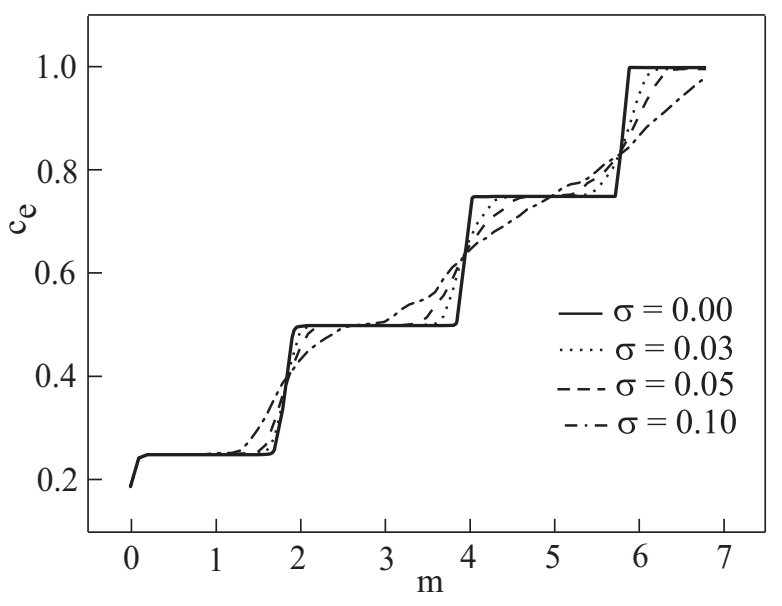

Fig. 4. An influence of disorder parameter $\sigma$ on low-temperature dependence $c_{e}(\mu)$ for $T=10^{-3} u\left(a_{0}\right), R_{0}=1$ and for host-lattice size $10 \times 10$.

\section{Phase transitions}

In this paragraph we will consider the phase diagram of the system. As far as Hamiltonian (3) has «antiferromagnetic» nature due to inter-electron repulsion, the most appropriative method is studying $c_{e}(T)$ dependence at different $\mu$. This dependence is analogue of magnetization $M$ over $T$ dependence at different values of external magnetic fields $H$ for magnetic systems. As it well known, the extremum point corresponds to phase transition temperature (Nèel point). Typical dependences $c_{e}(T)$ are presented in Fig. 6. One should pay attention to the structures of these curves. As was mentioned above, in

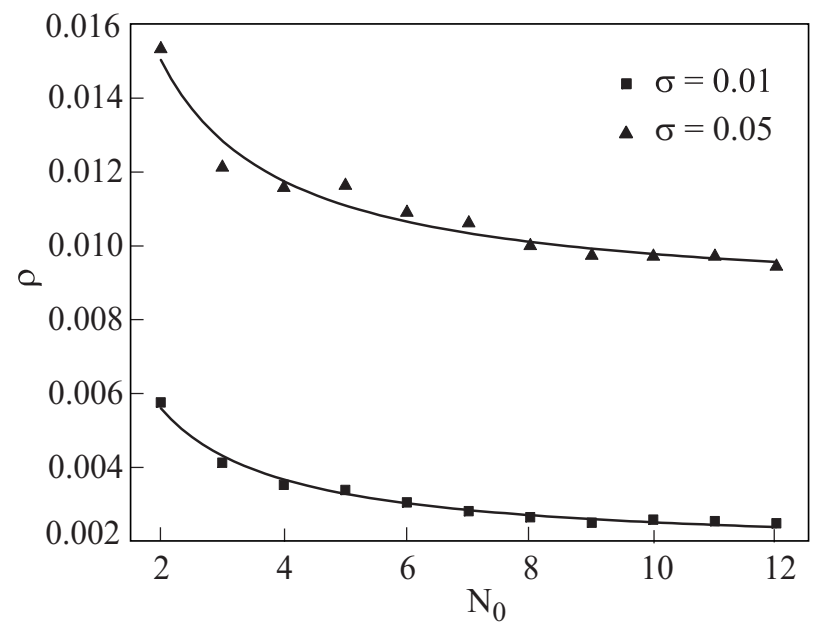

Fig. 5. Typical fluctuation $\rho$ as the function of initial configurations number $N_{0}$ for two values of disorder parameter $\sigma$ $(\sigma=0.01$ and $\sigma=0.05)$.

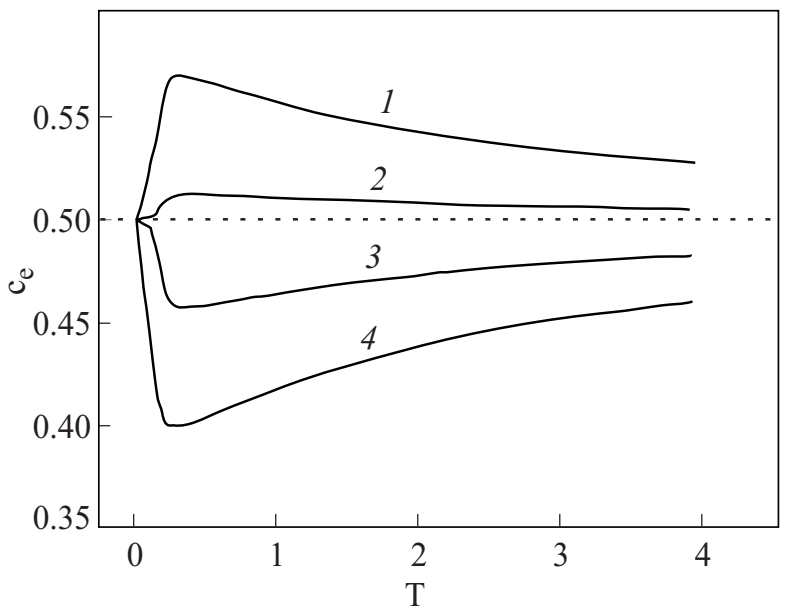

Fig. 6. Series of the dependences $c_{e}(T)$ corresponding to different $\mu$ values on ordered host-lattice $(\sigma=0)$. Host lattice size is $10 \times 10, R_{0}=1 . \mu: 3.5(1), 3(2), 2.5$ (3), 2 (4).

the simplest case of ordered host-lattice the system can be considered as spin assemble placed in both «internal field», produced by «exchange» interaction $u$ and «external field» conditioned by $\mu$. These fields have different sign and at some values of $\mu=2 \tilde{u}$ the total «field» changes the sign. Besides, there is additional term $-(\mu N / 2)$ in Hamiltonian. That is why at $\mu=2 \tilde{u}$ the dependence $c_{e}(T)$ changes their geometry. For $\mu<2 \tilde{u}$, $c_{e}(T)=1 / 2-M(T)$ and $c_{e}(T)=1 / 2+M(T)$ for $\mu>2 \tilde{u}$. Here $M(T)$ as a function which is analogous of $M(T)$ for antiferromagnets. For $\mu=2 \tilde{u}$ the total «field» equals zero and $c_{e}(T)=$ const $=1 / 2$.

Determining extremal points of $c_{e}(T)$ dependence for different $\mu$ one can plot the dependence $T_{c}(\mu)$, where $T_{c}$ is phase transition temperature. The results of numerical calculation for ordered $(\sigma=0)$ and disordered $(\sigma=0.05)$ host-lattices are presented in Fig. 7. One can see that for each region of $\mu$ in zero-temperature devil-staircase there is an «hat» in $T_{c}(\mu)$ dependence. It means that for any finite temperature $T$ change in $\mu$ parameter transforms the system from ordered phases (GWC) to disordered ones (like Wigner glass). The critical temperature is maximal in the vicinities of $\mu$ interval centers, tending to zero on the borders. This phenomenon explains a number of experiments in 2D semiconductor layers in external perpendicular electric fields. This field plays the role of $\mu$ because press out the volume electrons to $2 \mathrm{D}$ layer. For example, in experiments of $\mathrm{M}$. Pepper group [16,17] change in external field leaded to drastic change in electric conductivity in the layer. It means that in some interval of external electric fields (or in some $\mu$-regions in terms of proposed model) the GWC forms. The conductivity of the system decreased crucially due to localization of electrons. 


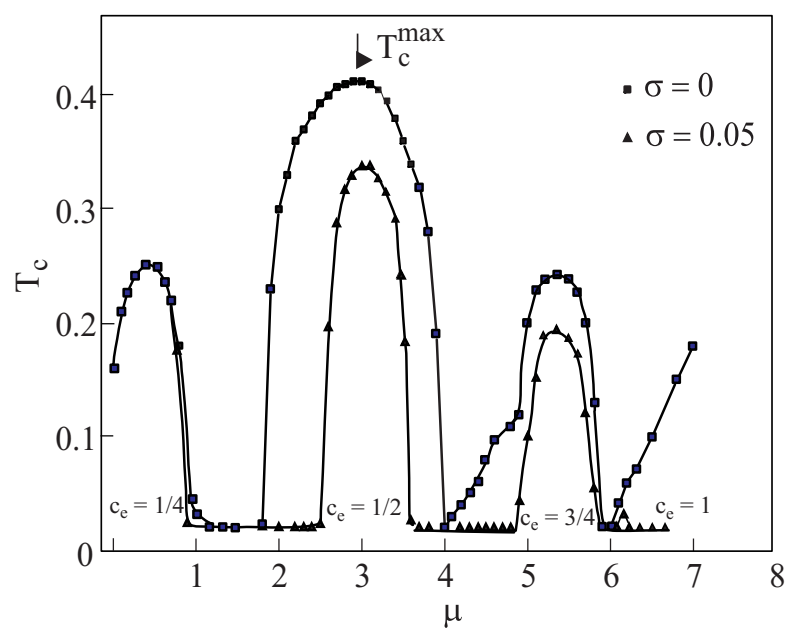

Fig. 7. The dependence $T_{c}(\mu)$ obtained for ordered $(\sigma=0)$ and disordered $(\sigma=0.05)$ host-lattice size $10 \times 10$ and $R_{0}=1$.

As it seen from Fig. 6, the heights of these «hats» decrease with disorder parameter $\sigma$ growth. This allows us to establish the critical temperature $T_{c}$ as the function of disorder parameter $\sigma . T_{c}^{\max }=T_{c}(\mu=3.1)$ and $R_{0}=1$ is presented in Fig. 8. The chemical potential $\mu=3.1$ corresponds to the maximum of $T_{c}$ for devil-staircase step with $c_{e}=1 / 2$. Solid line in this figure corresponds to the best fitting by the function $T_{c}=A_{0}\left(\sigma_{0}-\sigma\right)^{\alpha}$. Critical disorder parameter $\sigma_{0}=0.123$, power parameter $\alpha=0.38$.

\section{Results and discussion}

We have shown that the model of lattice gas with near-neighbor interaction leads to a rich thermodynamics. In spite of the simplicity of the model, it contains the most essential features of discrete 2D systems of such a sort. It is established that in low-temperature limit and weak host-lattice sites position disorder the system under consideration is generalized Wigner crystal. It means, in particular, that zero-temperature dependence of electron concentration $c_{e}$ as the function of chemical potential $\mu$ is fractal structure of devil staircase type. In this structure for each interval of $\mu$ there is a rational value of $c_{e}$ (so-called devil stair). This result fully confirms to preliminary investigations [1], which are valid, however, for concentration range $c_{e}<1 / 2$. Now, one can to assert that the generalized Wigner crystal formation takes place in whole concentration range $c_{e} \leq 1$.

It is clear, that at any finite temperature $T$ and disorder parameter $\sigma$ the devil stairs are «diffused». The ranges of stability and corresponding critical temperatures $T_{c}$ as the function of chemical potential and disorder parameter have been established. Besides, it is shown that change in chemical potential leads to successive transitions of the systems from ordered phases (generalized Wigner crys-

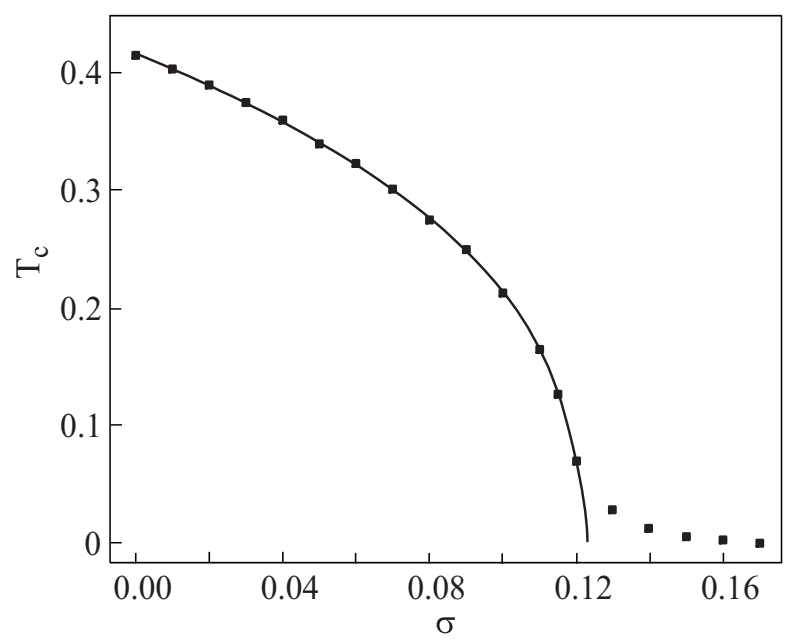

Fig. 8. The dependence $T_{\mathcal{C}}^{\max }=T_{\mathcal{c}}(\mu=3.1)$ as the function on $\rho$. Solid boxes are the results of numerical calculation. Solid line id the approximation by the function $T_{c}=A_{0}\left(\sigma_{0}-\sigma\right)^{\alpha}$. $\sigma_{0}=0.123, \alpha=0.38$.

tal) to disordered states like Wigner glass. This phenomenon may explain experimental results where small change in electron concentration leads to giant oscillation of conductivity in 2D plains (see, for example, [16-18]). Indeed, in this experiments the concentration of electrons (or holes) was varied by external electric filed applied perpendicular to $2 \mathrm{D}$ plain. As was mentioned above, this field plays the role of chemical potential. As the result, the conductivity in $2 \mathrm{D}$ plain changes drastically due to transitions from ordered phases to disordered states. It should be noted, that the question about conductivity properties of such systems is far from this paper frameworks. Indeed, we neglect the processes related to finiteness of $t$. Such an approximation is absolutely reasonable in «dielectric» (GWL) phase due to inequality $t<<\delta \varepsilon$ (see Introduction), but the finiteness of $t$ becomes important in the conductive («metallic») phase, where self-screening effects becomes strong.

It is planned to study the kinetic properties of such systems taking into account finiteness of $t$ by Monte-Carlo methods in near future.

1. A.A. Slutskin, V.V. Slavin, and H.A. Kovtun, Phys. Rev. B61, 14184 (2000).

2. P. Bak and R. Bruinsma, Phys. Rev. Lett. 49, 249 (1982).

3. Ya.G. Synay and S.Ye. Burkov, Russian Math. Surveys $\mathbf{3 8}$, 235 (1983).

4. J. Hubbard, Phys. Rev. B17, 494 (1978).

5. E.Y. Andrei, 2D Electron Systems on Helium and Other Substrates, Kluwer, New York (1997).

6. H. Nejoh and M. Aono, Appl. Phys. Lett. 64, 212803 (1995).

7. M.S. Bello, E.I. Levin, B.I. Shklovskii, and A.L. Efros, Sov. Phys. JETP 53, 822 (1981).

8. A.A. Slutskin, M. Pepper, and H.A. Kovtun, Europhys. Lett. 62 (5), 705 (2003). 
9. S. Fratini, B. Valenzuela, and D. Baeriswyl, Los-Alamos, cond-mat/0209518; cond-mat/0302020; cond-mat/0309450.

10. J. Jedrzejewski and J. Miekisz, Los-Alamos, cond-mat/ 9903163

11. G. Parisi, J. Phys. A13, 1101 (1980).

12. E. Marinari and G. Parisi, Europhys. Lett. 19, 451 (1992).

13. B.A. Berg, U.E. Hensmann, and T. Celik, Phys. Rev. B50, 16444 (1994).

14. K. Huang, Statistical Mechanics, John Wiley \& Sons, Inc., New York-London (1963).
15. V.V. Slavin and A.A. Slutskin, Phys. Rev. B54, 8095 (1996).

16. M. Pepper, J. Phys. C12, L617 (1979).

17. A. Ghosh, M.H. Wright, C. Siegert, M. Pepper, I. Farrer, C.J.B. Ford, and D.A. Ritchie, Phys. Rev. Lett. 95, 066603 (2005).

18. M. Baenninger, A. Ghosh, M. Pepper, H.E. Beere, I. Farrer, P. Atkinson, and D.A. Ritchie, Phys. Rev. B72, 241311(R) (2005) 